\title{
Oriented Thiocyanate Anions at the Air-Electrolyte Interface and its Implications on Interfacial Water - A Vibrational Sum Frequency Spectroscopy Study
}

\author{
P. Viswanath* and H. Motschmann ${ }^{\dagger}$ \\ Max Planck Institute of Colloids and Interfaces, \\ Am Mühlenberg 1, 14424, Golm/Potsdam, Germany.
}

Supporting Information

*viswanath.padmanabhan@mpikg.mpg.de

${ }^{\dagger}$ Corresponding author. hubert.motschmann@mpikg.mpg.de Fax : +49 3315679202 


\section{Experimental}

\section{Materials}

The salt, potassium thiocyanate $(\mathrm{KSCN}>99 \%)$ is purchased from fluka (catalogue number - 60519) is found to contain some alkyl impurities which is directly visible in the VSFG spectra around 2800-3000 $\mathrm{cm}^{-1}$ region. We have dissolved the salt in Millipore ${ }^{\circledR}$ water (resistivity $>18.2 \mathrm{M}^{-1} \mathrm{~cm}^{-1}$ ) followed by aspiration of the surface. Further we carried out crystallization process using the same solution and dried it under vacuum. All the experiments were carried out for $1 \mathrm{M}$ concentration in a dish at a temperature of $\sim 22^{\circ} \mathrm{C}$ and the surface of the solution is aspirated before recording the spectra.

\section{Methods}

For VSFG experiment, we use Nd:YAG laser (Ekspla). The oscillator output energy is $550 \mu \mathrm{J}$. The maximum output energy for the fundamental beam $(1.064 \mu \mathrm{m})$ is about $50 \mathrm{~mJ}$. The pulse duration is about $27 \mathrm{ps}$ with a repetition rate of $10 \mathrm{~Hz}$. The tunable IR is generated from the $\mathrm{AgGaS}_{2}$ crystal by DFG process using part of $1.06 \mu \mathrm{m}$ laser and tunable near-IR radiation from optical parametric generator (BBO crystal) as inputs. This DFG stage generated tunable IR output of $\sim 6 \mathrm{~cm}^{-1}$ bandwidth. The IR pulse energies at $2.94 \mu \mathrm{m}$ amd $4.84 \mu \mathrm{m}$ are $\sim 230 \mu \mathrm{J}$ and $\sim 260 \mu \mathrm{J}$ respectively and the visible $(532 \mathrm{~nm})$ pulse energy is around $\sim 200 \mu \mathrm{J}$. The tunable IR and the visible laser beams are overlapped both spatially and temporally at the electrolyte surface with incidence angles being $54^{\circ}$ and $59^{\circ}$ respectively. SFG signal was recorded by averaging 200 shots per point for every $2 \mathrm{~cm}^{-1}$ for thiocyanate and $5 \mathrm{~cm}^{-1}$ for water range. The spectrum is normalised to the intensities of the incident IR and visible beams.

\section{Data fitting}

Briefly, the normalized sum frequency intensity is given by, $I_{s f g} \propto\left|\chi_{n r}^{(2)}+\chi_{r}^{(2)}\right|^{2}$, where, $\chi_{n r}^{(2)}$ is the complex non-resonant susceptibility and, $\chi_{r}^{(2)}=\sum_{q} \frac{A_{q}}{\omega_{I R}-\omega_{q}+i \Gamma_{q}}$, where, $A_{q}$ is the amplitude of the resonant vibration, $\omega_{q}$ is the resonant frequency of the $q$ th mode, and $\Gamma_{q}$ is the damping constant. Customary programmes written in python were used for non-linear least squares fitting including the constraint on the phase for water region $(+$ for bonded and - ve for free $-\mathrm{OH}$ ) and also for the orientation analysis.

The fitted parameters for the CN stretch of thiocyanate anion under different polarization are listed in the Table 1 and the influence of potassium thiocyanate on the interfacial water along with water vibrational features are provided in Table 2. 


\begin{tabular}{|c|c|c|c|c|c|}
\hline Polarization & $\chi_{n r}^{(2), \text { real }}$ & $\chi_{n r}^{(2), \text { imag }}$ & $A_{q}$ & $\omega_{q}\left(\mathrm{~cm}^{-1}\right)$ & $\Gamma_{q}\left(\mathrm{~cm}^{-1}\right)$ \\
\hline Ssp & $0.002668 \pm 0.000244$ & $-0.01314 \pm 0.000147$ & $0.43234 \pm 0.04148$ & $2060.86 \pm 1.41$ & $13.95 \pm 1.43$ \\
ppp & $0.007736 \pm 0.000384$ & $-0.006583 \pm 0.00053$ & $0.617367 \pm 0.02276$ & $2064.31 \pm 0.72$ & $23.54 \pm 0.73$ \\
sps & $0.003305 \pm 0.000148$ & $-0.0076 \pm 0.000179$ & $0.57637 \pm 0.01499$ & $2064.05 \pm 0.63$ & $21.87 \pm 0.70$ \\
pss & $-0.000264 \pm 0.000118$ & $-0.006019 \pm 0.00015$ & $0.48916 \pm 0.01442$ & $2068.19 \pm 0.74$ & $23.10 \pm 0.88$ \\
\hline
\end{tabular}

Table 1: Fitted parameters for the CN stretch of thiocyanate anion in $1 \mathrm{M} \mathrm{KSCN}$ solution.

\begin{tabular}{|c|c|c|c|c|}
\hline System & $\chi_{n r}$ & $A_{q}$ & $\omega_{q}\left(\mathrm{~cm}^{-1}\right)$ & $\Gamma_{q}\left(\mathrm{~cm}^{-1}\right)$ \\
\hline $\mathrm{H}_{2} \mathrm{O}$ & -0.0067 & 0.6121 & 3232.9 & 129.82 \\
& & 2.2047 & 3433.7 & 150.96 \\
& & 0.467 & 3710.6 & 20.66 \\
\hline KSCN & -0.0038 & 0.2539 & 3322.6 & 56.44 \\
& & 1.5578 & 3440.2 & 101.58 \\
& & 0.5413 & 3716.1 & 25.76 \\
\hline
\end{tabular}

Table 2: Fitted parameters for water and $1 \mathrm{M} \mathrm{KSCN}$ solution under ssp polarization.

\section{Orientation Analysis}

There exists several reports in the literature which uses intensity ratio [1] or polarization analysis [2] as a means to obtain orientation. Based on that one can simulate the sum frequency intensities with respect to orientation angle which can then be compared directly with the experimentally obtained values to deduce orientation using,

$$
I_{s f g} \propto\left|\chi_{e f f, i j k}^{(2)}\right|^{2}=\left|L_{i i}\left(\omega_{s f g}\right) \chi_{i j k}^{(2)} L_{j j}\left(\omega_{v i s}\right) L_{k k}\left(\omega_{I R}\right)\right|^{2}
$$

For isotropic liquid surface with random azimuth the effective nonlinear susceptibilities under the four polarization combinations are given by,

$$
\begin{aligned}
& \chi_{e f f, S S P}^{(2)}=\sin \left(\beta_{i r}\right) L_{y y}\left(\omega_{s f}\right) L_{y y}\left(\omega_{v i s}\right) L_{z z}\left(\omega_{i r}\right) \chi_{y y z} \\
& \chi_{e f f, P P P}^{(2)}=-\cos \left(\beta_{s f}\right) \cos \left(\beta_{v i s}\right) \sin \left(\beta_{i r}\right) L_{x x}\left(\omega_{s f}\right) L_{x x}\left(\omega_{v i s}\right) L_{z z}\left(\omega_{i r}\right) \chi_{y y z} \\
&+\sin \left(\beta_{s f}\right) \sin \left(\beta_{v i s}\right) \sin \left(\beta_{i r}\right) L_{z z}\left(\omega_{s f}\right) L_{z z}\left(\omega_{v i s}\right) L_{z z}\left(\omega_{i r}\right) \chi_{z z z} \\
&-\cos \left(\beta_{s f}\right) \sin \left(\beta_{v i s}\right) \cos \left(\beta_{i r}\right) L_{x x}\left(\omega_{s f}\right) L_{z z}\left(\omega_{v i s}\right) L_{x x}\left(\omega_{i r}\right) \chi_{y z y} \\
&+\sin \left(\beta_{s f}\right) \cos \left(\beta_{v i s}\right) \cos \left(\beta_{i r}\right) L_{z z}\left(\omega_{s f}\right) L_{x x}\left(\omega_{v i s}\right) L_{x x}\left(\omega_{i r}\right) \chi_{z y y} \\
& \chi_{e f f, S P S}^{(2)}=\sin \left(\beta_{v i s}\right) L_{y y}\left(\omega_{s f} L_{z z}\left(\omega_{v i s}\right) L_{y y}\left(\omega_{i r}\right) \chi_{y z y}\right.
\end{aligned}
$$




$$
\chi_{e f f, P S S}^{(2)}=\sin \left(\beta_{s f}\right) L_{z z}\left(\omega_{s f}\right) L_{y y}\left(\omega_{v i s}\right) L_{y y}\left(\omega_{i r}\right) \chi_{y z y}
$$

The Fresnel factors are given by,

$$
\begin{gathered}
L_{x x}(\omega)=\frac{2 n_{1}(\omega) \cos (\gamma)}{n_{1}(\omega) \cos (\gamma)+n_{2}(\omega) \cos (\beta)} \\
L_{y y}(\omega)=\frac{2 n_{1}(\omega) \cos (\beta)}{n_{1}(\omega) \cos (\beta)+n_{2}(\omega) \cos (\gamma)} \\
L_{z z}(\omega)=\frac{2 n_{2}(\omega) \cos (\beta)}{n_{1}(\omega) \cos (\gamma)+n_{2}(\omega) \cos (\beta)}\left(\frac{n_{1}(\omega)}{n^{\prime}(\omega)}\right)^{2}
\end{gathered}
$$

where, $\mathrm{n}^{6}(\omega)$ is the interfacial refractive index which for our case is taken as 1.15, $\mathrm{n}_{1}$ and $\mathrm{n}_{2}$ are taken as 1.0 and $1.35[3]$ and assumed to be the same for all frequencies. $\gamma$ is the refracted angle.

The triatomic, thiocyanate anion is linear with $C_{\infty v}$ symmetry and possess conjugated structure. Thus it differs from the spherical halides but possess similar chemical properties (pseudo halide). Three non-zero second order hyperpolarizabilities which contribute for this system are, $\beta_{a a c}=\beta_{b b c}$ and $\beta_{c c c}$ whose ratio $\left(r=\beta_{a a c} / \beta_{c c c}\right)$ is accessible through polarized Raman measurements which are related to the second order non-linear susceptibility as,

$$
\begin{gathered}
\chi_{y y z}=\frac{N_{s}}{2} \beta_{c c c}\left[(r+1)<\cos \theta>-(1-r)<\cos ^{3} \theta>\right] \\
\chi_{y z y}=\frac{N_{s}}{2} \beta_{c c c}\left[(1-r)\left(<\cos \theta>-<\cos ^{3} \theta>\right]\right. \\
\chi_{z z z}=\frac{N_{s}}{2} \beta_{c c c}\left[r<\cos \theta>+(1-r)<\cos ^{3} \theta>\right]
\end{gathered}
$$

Unlike the monolayer which are orientationally ordered, the ions are randomized in the solution and it is reasonable to use Gaussian distribution instead of delta distribution for the orientation function, $f(\theta)\left(\cos \theta\right.$ or $\left.\cos ^{3} \theta\right)$ which is of the form, $<f(\theta)>=$ $\frac{1}{\sigma \sqrt{(2 \pi)}} \int_{0}^{\pi} f(\theta) \exp \left(-\frac{\left(\theta-\theta_{0}\right)^{2}}{2 \sigma^{2}}\right) \sin \theta d \theta$.

We have simulated the polarization dependence of sum frequency intensities using the reported raman depolarization ratio $(\rho)$ for thiocyanate in the range $0.38-0.42$ [4], we have calculated $r$ using the equation, $\rho=3 / 4\left[1+\frac{5(2 r+1)^{2}}{4(r-1)^{2}}\right]^{-1}$ which is found to be either -1.68 or -0.0407 . We have chosen the latter value for our calculations in accordance with our intensity dependence for various polarization $\left(A_{p p p}>A_{s s p}\right)$ while using the former value results in wrong dependence. The parameters used for simulating the intensity ratio are given in Table 3 . 


\begin{tabular}{|c|c|c|c|}
\hline Parameters & SFG & Vis & IR \\
\hline$\omega\left(\mathrm{cm}^{-1}\right)$ & 20862 & 18797 & 2065 \\
$\theta_{\text {in }}$ (deg. $)$ & 58.5 & 59.0 & 54.0 \\
$n_{\text {air }}$ & 1.0 & 1.0 & 1.0 \\
$n_{\text {aqueous }}$ & 1.35 & 1.35 & 1.35 \\
$n_{\text {interface }}$ & 1.1569 & 1.1569 & 1.1569 \\
$L_{x x}$ & 1.0469 & 1.0526 & 1.0044 \\
$L_{y y}$ & 0.6662 & 0.6611 & 0.7045 \\
$L_{z z}$ & 0.7121 & 0.7078 & 0.7438 \\
\hline
\end{tabular}

Table 3: Parameters used for simulating the intensity ratios at the air-electrolyte interface in the $-\mathrm{SCN}$ stretch region. 


\section{References}

[1] Zhuang, X.; Miranda, P.B.; Kim, D.; Shen, Y.R., Phys.Rev.B, 1999, 59, 12632.

[2] Wang, H-F.; Gan, W.; Lu, R.; Rao, Y.; Wu, B-H., Int. Rev. Phys. Chem., 2005, 24, 191.

[3] Bostrom, M.; Williams, D.R.M.; Ninham, B.W., Biophys. Jour., 2003, 85, 686.

[4] Kato, T.; Takenaka, T., Molec. Phys., 1982, 46, 257. 